\title{
Níveis de Ansiedade, Estresse Percebido e Suporte Social em Pessoas que Vivem com HIV/Aids
}

\author{
Prisla Ücker Calvetti ${ }^{1}$ \\ Universidade La Salle \\ Grazielly Rita Marques Giovelli \\ Gabriel Jose Chitto Gauer \\ João Feliz Duarte de Moraes \\ Pontifícia Universidade Católica do Rio Grande do Sul
}

\begin{abstract}
RESUMO - O objetivo deste estudo foi avaliar níveis de ansiedade, estresse percebido e suporte social em pessoas com HIV/ Aids em tratamento antirretroviral. Participaram deste estudo transversal descritivo e correlacional 120 pessoas (idade média = 42 anos) em tratamento antirretroviral para HIV/Aids atendidas em serviços de saúde pública. Foram utilizados Inventário de Ansiedade de Beck, Escala de Estresse Percebido e Escala de Suporte Social para Pessoas Vivendo com HIV/Aids. Sugere-se a importância do suporte social como fator de proteção à saúde na adesão ao tratamento.
\end{abstract}

Palavras-Chave: psicologia da saúde, aids, ansiedade, stress, suporte social

\section{Levels of Anxiety, Perceived Stress and Social Support in People Living with HIV/AIDS}

\begin{abstract}
The objective of this study was to evaluate symptoms of anxiety and perceived stress, and social support in people living with HIV/AIDS in an antiretroviral treatment. The participants of this descriptive and cross-sectional study were 120 people (mean age $=42$ years) in antiretroviral treatment for HIV/AIDS in public health services. The following instruments were administered: Beck Anxiety Inventory (BAI), Perceived Stress Scale and Support Social Scale for People living with HIV/Aids. Social support is a factor of health protection on adherence to antiretroviral treatment. Social support is important since it is related to having access to health services and to information about the illness, feeling well about physical health, safety and to the environment where he/shethe patient lives.
\end{abstract}

Keywords: health psychology, acquired immune deficiency syndrome, anxiety, stress, social support

A Psicologia da Saúde, visando à interdisciplinaridade, considera um conjunto de contribuições profissionais, científicas e educacionais para a prevenção, o tratamento e a reabilitação de enfermidades. Tem por objetivo a promoção de saúde bem como a análise e melhoria dos serviços de saúde e estratégias de intervenção nas políticas públicas sanitárias (Matarazzo, 1982). No contexto da saúde pública, há três décadas, implementou-se, no Brasil, a política de atenção às pessoas vivendo com HIV/Aids, por meio da Coordenação Nacional de DST/Aids. O vírus da Imunodeficiência Humana (HIV) e a Síndrome da Imunodeficiência Adquirida (Aids) atualmente têm prevenção e tratamento. As pessoas que vivem com a doença podem usufruir de melhor qualidade de vida se aderirem ao tratamento antirretroviral (Calvetti, 2014).

A Aids é a manifestação clínica avançada decorrente de um quadro de imunodeficiência causado pelo vírus HIV. À medida que a infecção pelo vírus progride, o sistema imunológico torna-se mais fraco, e o paciente fica mais suscetível a infecções oportunistas. O sistema imunológico

1 Endereço para correspondência: Programa de Pós-graduação Stricto Sensu Mestrado Saúde e Desenvolvimento Humano, Universidade La Salle, Av. Victor Barreto 2288, Canoa, RS, Brasil. CEP: 92.010-000. E-mail: prisla.calvetti@gmail.com não consegue lutar contra as infecções e doenças: o corpo fica vulnerável e, como consequência, perde-se a capacidade de resposta do organismo diante de agentes como vírus, bactérias e outros microrganismos A Aids pode ser considerada uma doença crônica, para a qual não há cura, mas há tratamento. $\mathrm{O}$ indivíduo com HIV pode viver com o vírus por vários anos sem manifestar nenhum sinal ou sintoma da doença (Fraporti, 2015; Ministério da Saúde, 2013).

Apesar do advento do antirretroviral, que auxilia no aumento da qualidade e expectativa de vida, o HIV/Aids apresenta um impacto psicossocial nas pessoas infectadas o que acarreta novas implicações: revisão das perspectivas do futuro; necessidade de uma redefinição dos objetivos pessoais, da situação profissional e dos relacionamentos; reavaliação de expectativas, crenças e benefícios com relação ao tratamento; necessidade de fortalecimento dos vínculos afetivos, entre outros (Campos, César, \& Guimarães, 2009). Nesse contexto, podem ser observados, em alguns casos, sentimentos de ansiedade, depressão, ira, culpa, revolta (raiva), bem como alta incidência de estresse, obsessões e auto-observação. Alterações ocorridas no sistema nervoso central dos pacientes com HIV/Aids, associadas a ansiedade, depressão e estresse podem influenciar a evolução da doença, 
uma vez que contribuem para o aumento da vulnerabilidade biológica (Springer, Dushaj, \& Azar, 2012).

A terapia antirretroviral tem se mostrado relacionada a resultados positivos de eficácia, aumentando a sobrevida dos pacientes infectados. Entretanto, a não adesão ao tratamento tem se mostrado importante fator de preocupação de saúde pública. A adesão está associada ao modo como a pessoa integra o uso da medicação e hábitos de cuidado ao seu dia a dia (Remor, Milner-Moskovics, \& Preussler, 2007).

Diante dessa premissa, estudos apontam as relações entre as variáveis psicológicas e a percepção de qualidade vida, identificando fatores que propiciam o ajustamento à condição de enfermidade crônica e ao enfrentamento ativo diante da disponibilidade de tratamento (Gaviria, Quiceno, Vinaccia, Martínez, \& Otalvaro, 2009; Seidl, Zannon, \& Tróccoli, 2005). Evidencia-se a associação entre disponibilidade e satisfação com o suporte social, bem-estar psicológico e percepção positiva da qualidade de vida, salientando-se a importância do papel do suporte como moderador do estresse em contextos relacionados ao processo saúde-doença.

A fim de contribuir para as ações no campo da atenção às pessoas que vivem com HIV/Aids, o objetivo desta pesquisa foi avaliar os níveis de ansiedade, estresse percebido e percepção do suporte social em pessoas em tratamento antirretroviral.

\section{Método}

Trata-se de estudo quantitativo, transversal, de caráter descritivo e analítico. A amostra foi selecionada por conveniência e composta por 120 participantes adultos do sexo masculino e feminino, com idades entre 18 e 65 anos, diagnosticados como portadores de HIV/Aids em tratamento antirretroviral. Os dados foram coletados em dois serviços de saúde pública de referência na atenção às pessoas que vivem com HIV/Aids e em uma organização não-governamental do estado do Rio Grande do Sul.

Os instrumentos utilizados foram os seguintes:

- Inventário de Ansiedade de Beck: avalia a intensidade da ansiedade clínica (auto-informada) através de 21 itens pontuados com o valor mínimo de zero e o valor máximo de 63 , correspondendo a maior pontuação a um grau mais elevado de ansiedade (Beck, Epstein, Brown, \& Steer, 1988; Cunha 2001).

- Escala de Stress Percebido (PSS): A PSS possui 14 questões com opções de resposta que variam de zero a quatro. Possui itens com conotação positiva e negativa do estresse. O total da escala é a soma das pontuações dessas 14 questões e os escores podem variar de zero a 56 , sendo que quanto maior a pontuação maior o nível de estresse percebido (Luft, Sanches, Mazo, \& Andrade, 2007).

- Escala de Suporte Social para Pessoas Vivendo com HIV/AIDS: instrumento específico para pessoas soropositivas destinado à mensuração do suporte social percebido e da satisfação em relação ao mesmo. Foi originalmente construído e validado no Canadá pelos autores Renwick, Halpen, Rudman e Friedland (1999) e denominado Social Support Inventory for People who are Positive or Have Aids. No Brasil, foi validado por Seidl e Tróccoli (2006). É composto por 24 itens, com escala de resposta tipo Likert de cinco pontos, podendo ser auto-aplicado ou administrado mediante entrevista. Dividi-se em dois fatores: suporte emocional e instrumental.

O primeiro contato com os participantes foi realizado em dias de consulta médica, enquanto esperavam na sala de espera ou logo após o atendimento. A aplicação dos instrumentos foi feita individualmente, realizada por bolsistas de iniciação científica treinados, em uma sala reservada, durante aproximadamente uma hora. Também foram acessados os prontuários dos pacientes para a coleta dos dados biológicos: carga viral e CD4+. Quando identificado, durante a avaliação dos participantes, a necessidade de algum encaminhamento para atendimento psicológico foi disponibilizado o serviço de atendimento terapêutico de uma organização não-governamental do Rio Grande do Sul.

Em relação aos procedimentos para análise dos dados, as respostas aos instrumentos foram submetidas a análises descritivas de frequências, médias e desvio-padrão das variáveis e a análises inferenciais sobre a relação entre aspectos psicológicos e marcadores biológicos através da análise de correlação de Pearson. Foi utilizado o nível de significância de 5\%, sendo os dados computados e analisados no Statistical Package for Social Sciences (SPSS) versão 17.5.

Este estudo teve aprovação da Comissão Científica da Faculdade de Psicologia da Pontifícia Universidade Católica do Rio Grande do Sul (PUCRS) e do Comitê de Ética em Pesquisa da PUCRS (CEP 08/04228). Recebeu financiamentos pelo Edital MCT/CNPq 03/2008 - Ciências Humanas, Sociais e Sociais Aplicadas (processo 400325/2008-0) e pelo CEARGS/Universidade da CalifórniaSão Francisco - "International Clinical, Outcomes and Health Services Research and Training Award from the John E. Fogarty International Center of the United States National Institutes of Health' (5D43TW005799).

\section{Resultados e Discussão}

A tabela I mostra os dados sociodemográficos e a situação clínica dos 120 participantes desta pesquisa. Nota-se que a percepção de saúde é boa para a maioria dos participantes (76\%). Entre os problemas de ordem psicológica descritos diante da pergunta "se alguma coisa não vai bem consigo", foram relatados sinais de ansiedade, tristeza, medo, baixa autoestima e culpa. Entre os problemas relacionados à saúde física, foram mencionados lipodistrofia, problemas de visão, perna operada, mal-estar abdominal, hepatite, baixa imunidade, HIV.

A tabela II apresenta as médias e o desvio padrão dos sintomas de ansiedade, estresse percebido e suporte social. A tabela III aponta o coeficiente de correlação linear de Pearson entre sintomas de ansiedade, estresse percebido e suporte social.

Os resultados encontrados demonstram que estresse percebido e sintomas de ansiedade possuem uma correlação direta, sendo o suporte social inversamente correlacionado 
ao estresse percebido. Neste estudo, os achados evidenciados apontam correlação entre os aspectos psicossociais: ansiedade, estresse percebido e suporte social em pessoas que vivem com HIV/Aids. O estresse percebido e os sintomas de ansiedade possuem uma correlação direta, entretanto, a correlação entre o suporte social e o estresse mostra-se como inversa, quanto maior o suporte social que a pessoa tem, menor é o estresse vivenciado e vice-versa..

Os sistemas de suporte fazem parte de um âmbito mais amplo da vida das pessoas, trata-se das relações estabelecidas com a escola, o trabalho, centros religiosos ou instituições de saúde. Pode-se inferir, diante desse quadro, que o suporte social seja fator de proteção à saúde nesta população, uma vez que é percebido como o sentimento e a percepção de estima e pertencimento do indivíduo a uma rede social com direitos e deveres comuns. Diante da

Tabela 1. Caracterização da amostra dados sociodemográficos e clínicos $(N=120)$

\begin{tabular}{|c|c|c|}
\hline & $\%$ & $F$ \\
\hline \multicolumn{3}{|l|}{ Escolaridade } \\
\hline $1^{\mathrm{o}}$ incompleto & 10,9 & 13 \\
\hline $1^{\mathrm{o}}$ completo & 35,0 & 42 \\
\hline $2^{\circ}$ completo & 40,8 & 49 \\
\hline $3^{\circ}$ incompleto & 2,5 & 3 \\
\hline $3^{\circ}$ completo & 10,8 & 13 \\
\hline \multicolumn{3}{|l|}{ Sexo } \\
\hline Feminino & 49,2 & 59 \\
\hline Masculino & 50,8 & 61 \\
\hline \multicolumn{3}{|l|}{ Situação de trabalho } \\
\hline Trabalha & 42,9 & 51 \\
\hline Não trabalha & 57,1 & 69 \\
\hline \multicolumn{3}{|l|}{ Estado Civil } \\
\hline Solteiro & 56,7 & 68 \\
\hline Casado ou vivendo com companheiro & 15,0 & 18 \\
\hline Separado ou divorciado & 22,5 & 27 \\
\hline Viúvo & 5,0 & 6 \\
\hline \multicolumn{3}{|l|}{ Carga viral (cópias/ml) } \\
\hline <50 (indetectável) & 68,3 & 82 \\
\hline $51-400$ & 15,0 & 18 \\
\hline $401-30.000$ & 13,3 & 16 \\
\hline$>30.000$ & 3,3 & 4 \\
\hline \multicolumn{3}{|l|}{ Considera-se doente } \\
\hline Sim & 23,3 & \\
\hline Não & 76,7 & \\
\hline \multicolumn{3}{|l|}{ Alguma coisa não está bem consigo } \\
\hline Sinais/sintomas físicos & 14,5 & 17 \\
\hline Sinais/sintomas psicológicos & 24,8 & 30 \\
\hline Relações sociais & 2,4 & 3 \\
\hline Condição sócio-econômica & 1,6 & 2 \\
\hline Considera tudo bem & 56,7 & 68 \\
\hline
\end{tabular}

tendência à cronicidade, novos desafios em especial na esfera psicossocial são emergentes no intuito de diminuir situações de discriminação. A atenção à saúde das pessoas que vivem com HIV necessita ser pautada na interdisciplinaridade bem como no respeito às singularidades (Seidl \& Faustino, 2014). Portanto, as percepções de interações sociais com outros indivíduos podem aumentar a autoestima, a auto-valorização e a autoconfiança de pessoas que vivem com HIV/Aids, reduzindo a percepção de estigmatização (Springer, Dushaj, \& Azar, 2012; Simoni, Montoya, Huang \& Goodry, 2005).

$\mathrm{O}$ estresse percebido, os sintomas de ansiedade e o suporte social, trazidos em relevância nesta pesquisa, influenciam a qualidade de vida da pessoa que vive com HIV/Aids (Seidl et al., 2005, Seidl \& Faustino, 2014). Seguindo nessa direção, tais aspectos influenciam, também, a adesão ao tratamento antirretroviral, que, atualmente, é fator de preocupação significativa por parte dos programas de saúde pública. Os sintomas psicológicos negativos podem contribuir para a não-adesão à terapia antirretroviral levando à baixa imunidade, ao aumento da resposta virológica (Campos, Guimarães, \& Remien, 2008) e, consequentemente à progressão da Aids. Estudo salientou que pessoas com depressão, ansiedade ou pânico generalizado foram aproximadamente duas vezes mais não-aderentes que aquelas sem um transtorno psicológico (Tucker, Burnam, Sherbourne, Kung, \& Gifford, 2003). A relação entre sintomatologia psicopatológica, adesão ao tratamento e qualidade de vida Tabela 2. Apresentação das médias e desvio padrão dos sintomas de ansiedade, do estresse percebido e suporte social

\begin{tabular}{lcccc}
\multicolumn{1}{c}{ Variável } & \% & F & Média & $\begin{array}{c}\text { Desvio } \\
\text { Padrão }\end{array}$ \\
\hline Níveis de Ansiedade (BAI) & & & & \\
Mínimo & 49,2 & 59 & & \\
Leve & 26,7 & 32 & & \\
Moderado & 2,5 & 15 & & \\
Grave & 11,7 & 14 & & \\
Escore Total & & & 14,17 & 12,59 \\
Estresse Percebido (PSS) & & & & \\
Estresse percebido - fator 1 & & & 13,15 & 6,01 \\
Conotação positiva - fator 2 & & & 8,99 & 6,34 \\
Escore Bruto & & & 22,14 & 11,05 \\
Suporte Social & & & & \\
Emocional - fator 1 & & & 3,56 & 1,00 \\
Instrumental - fator 2 & & 3,41 & 0,99 \\
\hline
\end{tabular}

Tabela 3. Correlação entre sintomas de ansiedade, estresse percebido e suporte social ao tratamento de pessoas que vivem com HIV/Aids

\begin{tabular}{lc}
\multicolumn{1}{c}{ Variável } & $\begin{array}{c}\text { Escore bruto Estresse Percebido } \\
\boldsymbol{r}\end{array}$ \\
\hline Ansiedade total & $0,631^{* *}$ \\
Suporte social & \\
Emocional - Fator 1 & $-0,576^{* *}$ \\
Instrumental - Fator 2 & $-0,455^{* *}$ \\
\hline Nota. ${ }^{* *}$ A correlação é significativa ao nível de $1 \%$
\end{tabular}


na infecção por HIV/AIDS também foi evidenciada por Reis, Leancastre, Guerra e Remor (2010). Faz-se necessária, portanto, a atenção psicossocial às pessoas que vivem com HIV/Aids, considerando a cronicidade da doença bem como a estigmatização presente.

\section{Conclusão}

Diante dos resultados alcançados, conclui-se a importância do fortalecimento do suporte social como foco de recursos e implementação de programas de saúde, em um planejamento de cuidado integral por toda a equipe que acompanha as pessoas que vivem com HIV/Aids. É fundamental o investimento na atenção aos aspectos psicossociais dos pacientes, pois estes constituem fatores de suma relevância na qualidade de vida dessas pessoas e, consequentemente, na adesão ao tratamento medicamentoso. Os psicólogos e pesquisadores no contexto da saúde necessitam incorporar às suas práticas o fortalecimento do suporte social por meio da abordagem do tema nas intervenções realizadas, bem como por meio de ações que incluam a rede de apoio das pessoas que vivem com HIV/Aids. O modelo biopsicossocial da Psicologia da Saúde auxilia o entendimento e a implementação dessa prática.

\section{Referências}

Beck, A. T., Epstein, N., Brown, G., \& Steer, R. A. (1988). An inventory for measuring clinical anxiety: Psychometric properties. Journal of Consulting and Clinical Psychology, 58, 893-897.

Brasil. (2013). Manual técnico para diagnóstico de infecção pelo $H I V$. Brasília: Ministério da Saúde, Secretaria de Vigilância e saúde. Departamento de DST, AIDS e Hepatites Virais.

Calvetti, P. Ü., Giovelli, G. R. M., Gauer, G. J. C., \& Moraes, J. F. D. M. (2014). Psychosocial factors associated with adherence to treatment and quality of life in people living with HIV/ AIDS in Brazil. Brazilian Journal of Psychiatry, 63(1), 8-15.

Campos, L. N., Guimarães, M. D. C., \& Remien, R. H. (2008). Anxiety and depression symptoms as risk factors for nonadherence to antiretroviral therapy in Brazil. Aids Behavior, 18(6), 529-536.

Campos, L. N., César, C. C., \& Guimarães, M. D. C. (2009). Quality of life among HIV-infected patients in Brazil after initiation of treatment. Clinics, 64(9), 867-875.

Cunha, J.A. (2001). Manual da versão em português das Escalas Beck. São Paulo: Casa do Psicólogo.

Fraporti, C. (2015). Características sociodemográficas da população que busca o teste rápido como diagnóstico inicial do HIV em uma Unidade de Pronto-Atendimento (Dissertação de mestrado). Mestrado em Saúde e Desenvolvimento Humano, Centro Universitário La Salle, Canoas.

Gaviria, A. M., Quiceno, J. M., Vinaccia, S., Martínez, L. A., \& Otalvaro, M. C. (2009). Estrategias de afrontamiento y ansiedad-depresión en pacientes diagnosticados com vih/sida. Terapia Psicológica, 27(1), 5-13.
Luft, C. D. B., Sanches, S. O., Mazo, G. Z., \& Andrade, A. (2007). Versão brasileira da Escala de Estresse Percebido: Tradução e validação para idosos. Revista de Saúde Pública, 41(4), 606-615.

Matarazzo, J. (1982). Behavioural health's challenge to academic, scientific and professional psychology. American Psychologist, $37,1-14$.

Remor, E. A., Milner-Moskovics, J. \& Preussler, G. (2007). Adaptaçión brasileira do Cuestionario para la Evaluación de la Adhesión al Tratamiento Antiretroviral. Revista de Saúde Pública, 41(5), 685-694.

Renwick, R., Halpen, T., Rudman, D., \& Friedland, J. (1999). Description and validation of a measure of received support specific to HIV. Psychological Reports, 84, 663-673.

Reis, A. C., Leancastre, L., Guerra, M. P., \& Remor, E. (2010). Relação entre sintomatologia psicopatológia adesão ao tratamento e qualidade de vida na infecção do HIV e Aids. Psicologia: Reflexão e Crítica, 23(3), 420-429.

Seidl, E. M. F., \& Faustino, Q. M. (2014). Pessoas vivendo com HIV/Aids: Possibilidades de atuação da Psicologia. In E. M. F. Seidl \& M. C. O. S. Miyazaki (Eds.), Psicologia da Saúde: Pesquisa e atuação profissional no contexto de enfermidades crônicas (pp. 21-57). Curitiba: Juruá.

Seidl, E. M. F., Zannon C. M. L. C., \& Tróccoli B. T. (2005). Pessoas vivendo com HIV/AIDS: Enfrentamento, suporte social e qualidade de vida. Psicologia: Reflexão e Crítica, 18(2), 188-195.

Seidl, E. M. F., \& Tróccoli, B. T. (2006). Desenvolvimento de escala para avaliação do suporte social em HIV/AIDS. Psicologia: Teoria e Pesquisa, 22(3), 317-326.

Simoni, J. M., Montoya, H. D., Huang, B., \& Goodry, E. (2005). Social support and depressive symptomatology among HIVpositive women: The mediating role of selfesteem and mastery. Women \& Health, 41, 1-15.

Springer, S. A., Dushaj, A., \& Azar, M. M. (2012). The impact of DSM-IV mental disorders on adherence to combination antiretroviral therapy among adult persons living with HIV/ AIDS: A systematic review. AIDS and Behavior, 16(8), 2119-2143.

Tucker, J. S., Burnam, A., Sherbourne, C. D., Kung, F. Y., \& Gifford, A. L. (2003). Substance use and mental health correlates of nonadherence to antiretroviral medications in a sample of patients with human immunodeficiency virus infection. The American Journal of Medicine, 114, 573-580.

Recebido em 05.07.2013

Primeira decisão editorial em 22.01.2016

Versão final em 28.02.2016

Aceito em 25.04.2016 